\section{Influenza Vaccine Use in Nunavut: A Brief Overview of the Uptake Rates across the Regions}

Dear Editor,

Influenza is a common respiratory illness that affects millions of Canadians each year. ${ }^{1}$ For most healthy adults and children, infections are not severe, but for vulnerable and/or high-risk populations such as the elderly, young children, individuals living in Aboriginal communities and those suffering from chronic health conditions, influenza can cause serious health consequences and even death; approximately 4,000-8,000 Canadians die annually from influenza and/or related complications. ${ }^{2,3}$

Canadian Aboriginal people (e.g., First Nations people, Inuit, Métis) often face more serious health-related challenges than their non-Aboriginal counterparts, such as high rates of chronic and contagious diseases and shorter life expectancy. American statistics indicate that the influenza illness rate is 3-7 times higher among Alaskan Natives and Native Americans than in whites. ${ }^{4}$ Hence there is a need to ensure optimal prevention of influenza infection where possible in Aboriginal communities.

Universal influenza programs are known to limit influenza morbidity and mortality by: increasing the numbers of people directly protected from influenza by vaccination and indirectly through herd immunity, and supporting the development of expertise in vaccine procurement and delivery systems that may be needed for dealing with an influenza pandemic. ${ }^{5}$

In the Canadian territories, the Yukon has provided free influenza vaccines to all residents aged 18 or older since 1999; the Northwest Territories (NWT) has offered free influenza vaccines to its residents since 2003, and Nunavut introduced universal vaccination for those aged 6 months and up in the fall of $2005 .{ }^{3}$ Unfortunately, influenza vaccine effectiveness is difficult to quantify in northern Aboriginal communities (e.g., Nunavut, NWT) because influenza infections are typically not laboratory confirmed or often not well documented unless the patient is sent to a hospital. ${ }^{5}$

Has the universal influenza vaccine program led to an increased uptake in Nunavut?

In 2007-2008, community uptake of influenza vaccine across Nunavut varied widely across communities - ranging from $12 \%$ to $77 \%$ of eligible persons in all three regions (Figure 1). Furthermore, the variation continued in the 2008-2009 portion of the program (Figure 1) with overall immunization rates (0-60\%) worse than the previous season.

\section{Why the wide variation from community to community?}

In non-Aboriginal communities in the south, influenza vaccine uptake is influenced by a range of factors, including attitudes toward vaccination; perception of influenza risk and vaccine efficacy; frequency of health care visits; and most importantly, atti-
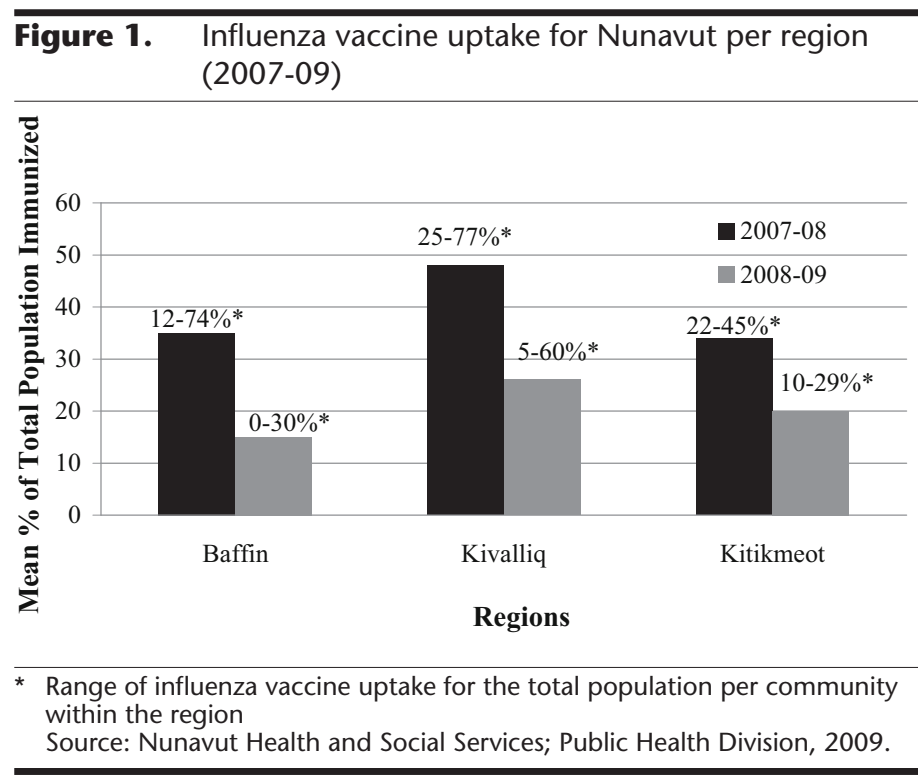

tudes of the health care providers. ${ }^{4}$ In the north, where access to care choices is limited in range, the attitude of the care provider may be especially influential.

\section{How might influenza vaccine uptake rates be improved in Nunavut?}

The first step will be to determine the attitudes and beliefs of the health care providers running the influenza vaccine program in each community and contrast these with the uptake rates. If a correlation is found, then efforts are needed to increase the education and skills of local health care providers with regard to optimization of influenza vaccine uptake. Given the high cost of medical evacuation for complicated influenza cases, this study needs to be done sooner than later.

Audrey A. Steenbeek, PhD, RN

School of Nursing, Dalhousie University, Halifax, NS

Isaac Sobol, MD, MPH

Nunavut Health \& Social Services, Iqaluit, NU

Noni E. MacDonald, MD, MSc

Dept. of Pediatrics, Dalhousie University, IWK Health Centre, Halifax, NS

\section{REFERENCES}

1. Health Canada: Diseases and conditions. Available online at: http://www.hcsc.gc.ca/dc-ma/influenza/index-eng.php (Accessed January 29, 2009).

2. Kwong JC, Rosella LC, Johansen H. Trends in influenza vaccination in Canada, 1996/1997 to 2005. Health Reports 2007;18(4):1-11.

3. Public Health Agency of Canada: Influenza. Available online at: http://www.phac-aspc.gc.ca/influenza/ (Accessed January 29, 2009).

4. Zimmerman RK, Tabbarah M, Nowalk MP, Raymund MR, Jewell IK, Hyg MS, et al. Predictors of pneumococcal polysaccharide vaccination among patients at three inner-city neighborhood health centers. Am J Geriatric Pharm 2005;3(3):149-59.

5. Kwong JC, Stukel TA, Lim J, McGeer AJ, Upshur RE, Johansen H, et al. The effect of universal influenza immunization on mortality and health care use. PLOS Medicine 2008;5(10):1440-52. 